\title{
Investigation of health anxiety and its related factors in nursing students
}

\author{
This article was published in the following Dove Press journal: \\ Neuropsychiatric Disease and Treatment \\ 7 July 2014 \\ Number of times this article has been viewed
}

\section{Yuqun Zhang ${ }^{1,2}$ \\ Yueqiu Zhao ${ }^{3}$ \\ Shengqin Mao' \\ Guohong $\mathrm{Li}^{4}$ \\ Yonggui Yuan'}

'Department of Psychosomatics and Psychiatry, Affiliated Zhongda Hospital, Medical School of Southeast University, Nanjing, People's Republic of China; ${ }^{2}$ Nursing Faculty of Southeast University, Nanjing, People's Republic of China; ${ }^{3}$ Nanjing Health School, Nanjing, People's Republic of China; ${ }^{4}$ Nursing Department, Affiliated Zhongda Hospital, Medical School of Southeast University, Nanjing, People's Republic of China
Correspondence:Yonggui Yuan Department of Psychosomatics and Psychiatry, Affiliated Zhongda Hospital, Medical School of Southeast University, No. 87 Dingjiaqiao, Gulou District, Nanjing, 210009 jiangsu Province, People's Republic of China Tel/fax +86258327 2160 Email yygylh2000@sina.com
Objective: To explore health anxiety in a sample of nursing students to determine the relationships between health anxiety and life satisfaction, personality, and alexithymia.

Methods: Two thousand and eighty-six nursing students in junior college, which were divided into five groups, were evaluated by questionnaires, including the Life Satisfaction Scales Applicable to College Students, the Chinese version of the Short Health Anxiety Inventory, the Toronto Alexithymia Scale (TAS-20), and the Eysenck Personality Questionnaire.

Results: The mean age, whether the individual was an only child, residence (urban or rural), and were significantly different between the groups. The self-assessment scores were also significantly different between the groups. The Short Health Anxiety Inventory total score and the factor of fearing the likelihood of becoming ill were significantly negatively correlated with the Life Satisfaction Scales Applicable to College Students total score and its two factors, but were significantly positively correlated with psychoticism, neuroticism, and TAS-20 total scores and its scores of the three TAS-20 factors. The negative consequence scale of Short Health Anxiety Inventory was not significantly correlated with externally oriented thinking, but was significantly negatively correlated with extraversion. A hierarchical multiple regression analysis indicted that objective satisfaction, subjective satisfaction, neuroticism, and the three factors of TAS-20 were predictors of health anxiety.

Conclusion: Health anxiety was correlated with life satisfaction, personality, and alexithymia in junior college nursing students. Subjective and objective satisfaction, neuroticism, and the identification and expression of emotions may be predictors of health anxiety in nursing students.

Keywords: life satisfaction, personality, alexithymia

\section{Introduction}

Health anxiety refers to the negative interpretation of and fears about the meaning of both ordinary and unusual bodily sensations. ${ }^{1}$ Research suggests that health anxiety is a dimensional construct characterized by a lack of concern about one's health at one end of a continuum and excessive anxiety at the other end. ${ }^{2}$ In its severe form, health anxiety has a significant long-term impact on the patient's self-rated functioning related to mental and physical health and on health care costs. ${ }^{3}$ A previous study has suggested that the prevalence of health anxiety was approximately 5\% in 2013 in the general population. ${ }^{4}$ Tyrer et $\mathrm{al}^{5}$ found that abnormal health anxiety is common and a significant problem in individuals attending medical clinics; the prevalence levels varied by clinic, with neurology having the highest prevalence $(24.7 \%)$, followed by respiratory medicine $(20.9 \%)$, gastroenterology $(19.5 \%)$, cardiology $(19.1 \%)$, and endocrinology (17.5\%). If patients' health worries are addressed at an early stage by physicians, repeated consultations and medical examinations may be prevented, and it could also prevent the development of more severe health anxiety that requires intensive intervention. ${ }^{3}$ 
The life satisfaction of students includes their academic record, interpersonal relationships, appearance, physical condition, economic condition, and total satisfaction for life. A previous study suggested that high-performing students may base their self-worth on feedback from their academic performance; ${ }^{6}$ thus, the academic record may impact the mental health of students. Interpersonal relationships are an important aspect of life satisfaction; in patients with borderline personality disorder, suicide threats are often related to emotions connected with interpersonal relationships. ${ }^{7}$ In their study of relationship satisfaction and depressive symptoms in emerging adults, Whitton and Kuryluk proposed that lower relationship satisfaction was associated with higher concurrent depressive symptoms and a heightened risk of future symptoms and diagnoses of depression. ${ }^{8}$ Thus, interventions that improve relationships, support systems, and family functioning may reduce the negative impact of stressors. ${ }^{9}$ Richards et al suggested that psychosocial factors and demographics were predictors of the development of appearance-related distress and compromised the psychological well-being of patients with ptosis. ${ }^{10}$ Therefore, appearance is an important aspect of health anxiety. Life satisfaction not only involves the above factors, but also focuses on the physical condition. A previous study that investigated the physical and mental health perspectives of first-year students found that, in the previous 6 months, physical conditions were reported, with the most common conditions being fatigue (56\%), frequent headaches (26\%), and allergies (24\%). ${ }^{11}$ Since ancient times, social scientists have known that social class is a profound dimension of social life. Rich individuals and poor individuals differ over a diverse array of domains that include aesthetic preferences, language, physical health, subjective well-being, and cognitive performance. ${ }^{12}$ Therefore, psychological problems caused by an individual's economic condition are worth exploring.

A number of alternative dimensional models of both normal and pathological personality traits have been developed. In 2005, Widiger and Simonsen ${ }^{13}$ identified five shared broad domains of personality traits: emotional dysregulation versus emotional stability; extraversion versus introversion; antagonism versus compliance; constraint versus impulsivity; and unconventionality versus closedness to experience. Longitudinal studies of the effects of psychoticism on young individuals are extremely limited and largely confined to studies of antisocial behaviors rather than emotional wellbeing. ${ }^{14}$ While current evidence suggests that, although psychoticism predicts poor adjustment and personality disorders, it does not predict psychosis. ${ }^{15}$ Furthermore, neuroticism was a reaction to the clinical symptoms of some diseases, but was not an etiological factor. ${ }^{16}$ While neuroticism is a more general construct related to negative affect, there are certain dysfunctional cognitions that are specifically related to pain. ${ }^{17}$

In 1970, Nemiah and Sifneos ${ }^{18}$ described alexithymia as a syndrome characterized by a deficit in the experiences and expression of feelings and a paucity of imaginable experience, which results in a style of thought devoid of fantasy and metaphor. Alexithymia is now considered a dimensional personality trait that refers to a cluster of deficits in the recognition, differentiation, and verbalization of emotions. ${ }^{19}$ The alexithymia construct comprises five components: 1) difficulty in identifying and describing subjective feelings; 2) difficulty in distinguishing between feelings and bodily sensations of emotional arousal; 3 ) reduced imaginative capacities, as evidenced by a paucity of fantasies; 4) an externally oriented thinking style, consisting of a tendency to focus on external events rather than inner experiences and to describe facts and actions without affective involvement; and 5) social conformity. ${ }^{20}$ The 20 -item Toronto Alexithymia Scale (TAS-20) assesses three of the components (1, 2, and 4). ${ }^{21}$ Zunhammer et $\mathrm{al}^{22}$ found that neuroticism, alexithymia, trait anxiety, and depression explained the variance in somatization at baseline. Their results showed that nursing students' concerns related to health and behaviors would be affected by alexithymia. Alexithymia is found in up to $10 \%$ of the general population and is associated with a lower quality of life. ${ }^{23}$ Alexithymia is a major risk factor for a range of medical and psychiatric problems.

A variety of factors are considered to be important in health anxiety. These factors include personality and mood characteristics and the potential for adaptation, including the ability to withstand emotional and physical distress. ${ }^{24}$ In addition to the above factors, other factors are also worthy of attention. Reiser et $\mathrm{al}^{25}$ demonstrated that adverse childhood experiences were predictive of health anxiety in adulthood. Physical diseases in childhood, higher rates of psychological and psychosocial problems in childhood, and experiences with familial illness are also factors that were found to influence health anxiety. ${ }^{26}$ Moreover, young adults reported higher levels of health anxiety compared with older adults. ${ }^{27}$ The negative factors of health anxiety not only included childhood experiences and age, but also health beliefs, the process of perceived fear, and safety behavior. ${ }^{28}$ In addition, objective factors can also impact health anxiety; for example, Sunderland et $\mathrm{al}^{4}$ in a study regarding health anxiety in Australia, found that age was a significant predictor 
in the model for both lifetime and current prevalence. The relationship between health anxiety and the quality of life, the expression of feelings, and the individual's personality have not been thoroughly researched.

College students are moving into and through a major developmental period of transition. As they grapple with increased academic, personal, social, and moral pressures in their lives, these stressors may lead to increased anxiety, loneliness, depression, hopelessness, headaches, sleep disturbances, colds, and, sometimes, suicidal ideations. ${ }^{29} \mathrm{With}$ regards to medical college students, reports indicate that medical school stress and factors related to the individual student, such as personality and previous mental health problems, contribute to mental health deterioration. ${ }^{30}$ Health anxiety is also an important aspect that affects college students' lives and studies. With regards to nursing students, the literature regarding mental health distress has focused on academic and personal sources of stress and coping efforts, with an emphasis on the stress and anxiety associated with clinical practice. ${ }^{31}$

The purpose of the present study was to explore the health anxiety and related factors of Chinese nursing students. The present study had two main objectives. The first objective was to examine the relationship between health anxiety and nursing students' life satisfaction, alexithymia, and personality through the Short Health Anxiety Inventory (SHAI), the Life Satisfaction Scales Applicable to College Students (CSLSS), the TAS-20, and the Eysenck Personality Questionnaire (EPQ). The hypotheses for the first objective were that the health anxiety of nursing students was negatively correlated with life satisfaction and positively correlated with alexithymia and personality. The second objective was to investigate the factors that influenced health anxiety in this study, which were verified by hierarchical regression analyses.

\section{Methods}

\section{Participants}

Two thousand and eighty-six junior college nursing students participated in this study. The students were divided into five groups according to their level of study (grade 1: $n=369$, mean age $16.60 \pm 0.71$ years, $2.79 \%$ male; grade $2: \mathrm{n}=475$, mean age $17.49 \pm 0.78$ years, $3.26 \%$ male; grade $3: n=298$, mean age $18.65 \pm 0.88$ years, $1.02 \%$ male; grade $4: \mathrm{n}=446$, mean age $19.93 \pm 0.75$ years, $3.48 \%$ male; grade $5: \mathrm{n}=498$, mean age $20.84 \pm 0.80$ years, $2.68 \%$ male). The age ranged from 16 to 24 years. The prevalence of females was greater compared with males because of the profession. Grade 3 had the highest percentage of females, at $98.99 \%$. Grade 4 had the highest percentage of only children, at $79.82 \%$. Grade 5 had the lowest percentage of individuals living in a city residence, at $48.59 \%$.

\section{Demographics}

Age, sex, being an only child, and residence (urban or rural) were selected as the demographics in our study. Being an only child and residence were related to economic status, and economic status is one part of life satisfaction. Different growth environments (eg different economic status, different geographical factors which could influence the growth etc) also affects personality and emotion.

\section{Measures SHAI}

The SHAI is an 18-item measure that assesses three aspects of health anxiety: illness likelihood, body vigilance, and the perceived negative consequences of becoming ill. ${ }^{32}$ Salkovskis et a $\mathrm{l}^{32}$ clarified two factors, which correspond to 1) the feared likelihood of becoming ill (Illness Likelihood [IL] subscale), and 2) the feared negative consequences of becoming ill (Negative Consequences [NC] subscale). Each item of the SHAI consists of four statements rated from "I do not" (0) to "I spend most of my time" (3). The total score ranges from 0 to 54 . The IL subscale contains items 1 to 14 , and the $\mathrm{NC}$ subscale contains items 15 to 18 . In consideration of the construct of health anxiety, cut-off scores were not used in the original article. The SHAI is a measure that is sensitive to both mild and more severe forms of health anxiety and is suitable for use in both medical and nonmedical samples. ${ }^{33}$ The SHAI has demonstrated adequate-to-excellent internal consistency in undergraduate students and strong construct validity. ${ }^{34}$ In our study, the Chinese version of the SHAI full scale has demonstrated good internal consistency (alpha $=0.864)$ and test-retest reliability $(r=0.562)$.

\section{CSLSS}

Wang ${ }^{35}$ developed the CSLSS on the basis of Campbell et al's scale of well-being. The CSLSS is a six-item measure that assesses the life quality of college students. It contains two factors: objective satisfaction ([OS] items 1-5) and subjective satisfaction ([SS] item 6). Each item consists of seven points that are rated from "very satisfactory" (7) to "never satisfactory" (1). Items 1,3 , and 5 are scored from 7 to 1 , whereas items 2,4 , and 6 are scored using a reverse scale. The total score ranges from 6 to 42 . The Chinese version of the CSLSS has demonstrated good internal consistency and test-retest reliability. Wang ${ }^{35}$ tested the correlation between 
each OS item and the OS total scores and found that all correlation coefficients were above 0.45 . The correlation coefficients of the OS total score and the SS total score, the OS total score and the CSLSS total score, and the SS total score and the CSLSS total score were $0.43,0.74$, and 0.93 , respectively. The test-retest reliabilities of each item were 0.72 (item 1), 0.75 (item 2), 0.69 (item 3), 0.84 (item 4), 0.82 (item 5), and 0.78 (item 6).

\section{TAS-20}

The TAS-20 is a 20-item questionnaire that asks participants to rate the degree to which they agree with each statement on a 5-point Likert-type scale that ranges from "never true" (1) to "very often true" (5). It contains three factors: difficulties identifying and distinguishing among feelings (TAS-F1), difficulties describing or communicating feelings (TAS-F2), and externally oriented thinking (TAS-F3). TAS-F1 contains items 1, 3, 6, 7, 9, 13, and 14. TAS-F2 contains items 2, 4, 11, 12, and 17. TAS-F3 contains items 5, 8, 10, 15, 16, 18, 19, and 20 . It is worth noting that the calculation of items $4,5,10,18$, and 19 use a reverse scale. The TAS-20 total score is the sum of TAS-F1, TAS-F2, and TAS-F3. The Chinese version of the TAS-20, which contains three factors, demonstrated good internal consistency (alpha $=0.645,0.630$, and 0.581 , respectively) and test-retest reliability ( $r=0.782,0.687$, and 0.893 , respectively) when it was translated by Yuan et al in $2003 .{ }^{36}$

\section{EPQ}

The EPQ is an 88-item questionnaire designed to measure personality dimensions or traits in a yes/no format. The EPQ assesses three basic personality dimensions (neuroticism, extraversion, and psychoticism), as well as truthfulness. ${ }^{38}$ Neuroticism $(\mathrm{N})$ reflects the stability of the emotion, with a higher score representing the most instability. "Extraversion" (E) reflects an introvert versus extrovert personality, with a higher score indicating an extrovert personality. "Psychoticism" (P) is referred to as "tough-mindedness"; the $\mathrm{P}$ dimension is viewed as an "unspecific vulnerability" in which individuals are predisposed to varying degrees of psychosis or a continuum that ranges from altruistic and empathic tendencies at one end to impulsive, aggressive, and other "near-psychotic" states at the other end. ${ }^{14}$ "Truthfulness" (L) reflects the level of concealment of the individual's objectives. The Chinese version of the EPQ, which was revised by Chen in 1983, has demonstrated good internal consistency (alpha $=0.68-0.81$ depending on the subscale) and test-retest reliability ( $r=0.67-0.92$ depending on the subscale). ${ }^{37}$ We calculated the EPQ scores using psychological measurement software (Psychological test software system, version number 5.0 [Shanghai Huicheng Company, Shanghai, People's Republic of China]) into which we imported every item the students chose and then obtained the scores of the four parts.

\section{Procedure}

The participants in this study were adult volunteer nursing students. Two thousand and eighty-six nursing students were divided into five groups according to grade. Students filled out the paper-based questionnaires anonymously in groups in their classrooms, and the entire procedure took approximately 20-30 minutes. The students did not receive an educational or other reward for their participation. The study procedure was approved by the ethics committee of Zhongda Hospital, which is affiliated with Southeast University, Nanjing, People's Republic of China.

\section{Analyses}

To complete the analyses, the Predictive Analytics Software (PASW) Statistics 18 package was used (IBM Corporation, Armonk, NY, USA). The categorical variables, including sex, being an only child, and residence, were compared by means of the chi-square test. One-way analysis of variance was employed to compare the continuous variables, such as age, between the groups. Pearson correlations were conducted to assess the relationships between health anxiety and satisfaction of life, alexithymia, and personality. With the SHAI total score as the dependent variable, a hierarchical linear regression model was performed. For all statistical analyses, differences were considered significant at $P<0.05$.

\section{Results \\ Demographics of the participants}

The participants' demographics are presented in Table 1. The ages in the five grades were significantly different (any two grades compared with each other, $P<0.001$ ). The results suggested that there was no statistically significant difference in sex between the five grades. Limited by the profession, females were clearly more prevalent than males. Grade 3 had the highest percentage of females. More than $70 \%$ of the nursing students were the only child in their families. Whether the participants were the only child in their families was significantly different between the groups. Approximately 50\% of the students lived in the city for each group.

\section{Comparisons of the measures}

The participants' scores are presented in Figures 1 to 4 . The SHAI total score was the sum of the SHAI-IL and the SHAI-NC. The SHAI total scores of grade 2 and grade 5 
Table I Main demographic variables among five grades of junior college students

\begin{tabular}{|c|c|c|c|c|c|c|}
\hline \multirow{2}{*}{$\begin{array}{l}\text { Demographic } \\
\text { variable }\end{array}$} & \multicolumn{5}{|c|}{ Junior college students } & \multirow[t]{2}{*}{$\chi^{2} / F$} \\
\hline & Grade I $(\mathbf{N}=369)$ & Grade $2(N=475)$ & Grade $3(N=298)$ & Grade $4(N=446)$ & Grade $5(N=498)$ & \\
\hline Mean age (SD), years & $16.60(0.71)$ & $17.49(0.78)$ & $18.65(0.88)$ & $19.93(0.75)$ & $20.84(0.80)$ & $2,148.422 * * *$ \\
\hline Sex (female, \%) & 97.29 & 96.84 & 98.99 & 96.64 & 97.39 & 4.416 \\
\hline Only child (yes, \%) & 77.24 & 77.47 & 72.48 & 79.82 & 72.69 & $9.468^{*}$ \\
\hline $\begin{array}{l}\text { Urban versus rural } \\
\text { (urban, \%) }\end{array}$ & 68.56 & 65.26 & 59.06 & 60.76 & 48.59 & $43.976 * * *$ \\
\hline
\end{tabular}

Notes: $* P<0.05$; *** $P<0.00$ I.

Abbreviation: SD, standard deviation.

were significantly higher compared with the other groups: $F(4,2,081)=4.040, P<0.01$ (Figure 1A). The SHAI-IL indicates the illness likelihood, and the scores showed similar results compared with the SHAI total score: $F(4,2,081)=3.449$, $P<0.01$. Moreover, the scores of grade 5 were significantly higher compared with grade $3(P<0.05)$ (Figure 1B). The SHAI-NC indicates fearing the negative consequences of becoming ill, and the scores of grade 1 were significantly lower compared with the other groups: $F(4,2,081)=5.325$, $P<0.001$. In addition, the scores of grade 5 were significantly
A

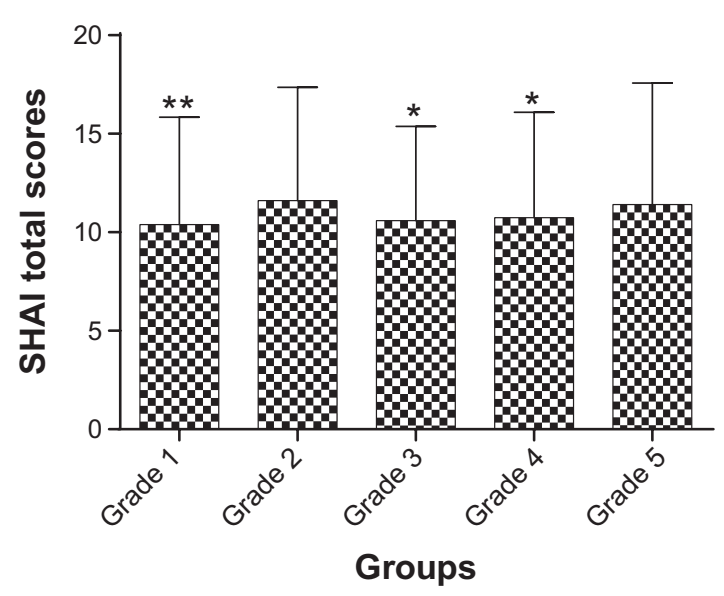

B

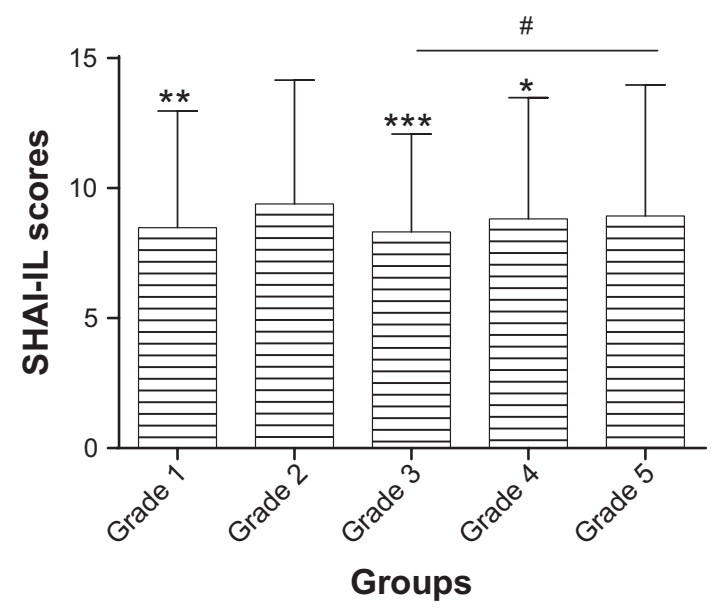

C

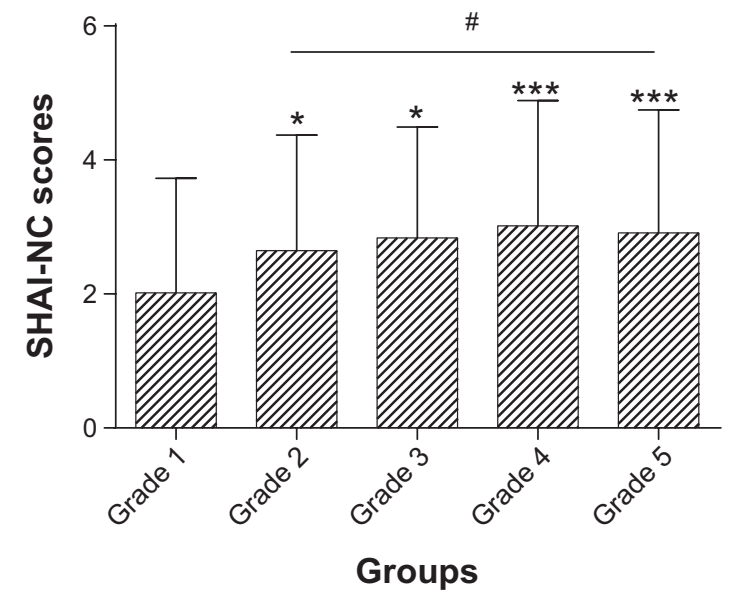

Figure I Comparisons of SHAI total scores and two subscale scores among five groups.

Notes: (A) SHAl total scores. SHAI total scores of grade 2 and grade 5 compared to other grades, respectively, $* P<0.05$; $* * P<0.01$. (B) SHAI-IL scores. SHAI-IL scores of grade 2 compared to other grades $* P<0.05$; $* * P<0.0$ I; $* * * P<0.00 \mathrm{I}$. SHAI-IL scores of grade 3 compared to grade 5 , ${ }^{\# P<0.05}$. (C) SHAI-NC scores. SHAI-NC scores of grade I compared to other grades, ${ }^{*} P<0.05$; ${ }^{* * * P}<0.00 \mathrm{I}$. SHAI-NC scores of grade 2 compared to grade 5 , ${ }^{*} P<0.05$.

Abbreviations: SHAI, Short Health Anxiety Inventory; SHAI-IL, SHAI Illness Likelihood subscale; SHAI-NC, SHAI Negative Consequences subscale. 
higher compared with grade $2(P<0.05)$ (Figure 1C). Grade 5 had significantly higher CSLSS total scores compared with the other grades: $F(4,2,081)=5.563, P<0.001$ (Figure 2A); a similar increase was found for the CSLSS-OS: $F(4,2,081)=5.167, P<0.001$ (Figure 2B) and the CSLSS-SS: $F(4,2,081)=2.838, P<0.05$ (Figure 2C). The EPQ reflects the personality of the subjects. In our study, the EPQ-E scores (Figure 3A) of grade 2 and grade 3 were significantly lower compared with the other groups: $F(4,2,081)=6.621$, $P<0.001$, but there was no difference between them. The EPQ-P scores (Figure 3B) of grade 1 and grade 2 were significantly higher compared with the other groups:
$F(4,2,081)=15.659, P<0.001$. The EPQ-N scores (Figure 3C) of grade 1 were significantly higher compared with the other groups: $F(4,2,081)=7.579, P<0.001$, and the scores of grade 4 were significantly higher compared with grade 2 : $(P<0.01)$. The EPQ-L scores (Figure 3D) reflect the students' truthfulness, and there was a difference between grade 1 and grade 4 , but the scores did not vary widely. Through the analysis of the three factors of the EPQ, we determined that the grade 1 students were more excited and more emotionally unstable. The TAS-20 contains three factors that predominately focus on emotions. The TAS-20 total scores (Figure 4A) of grade 1 were significantly higher compared with the other groups:
A

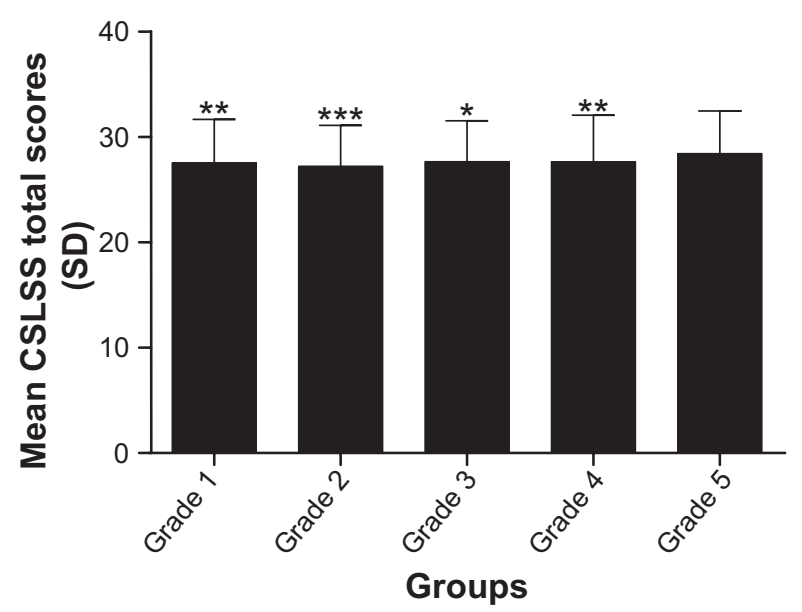

B

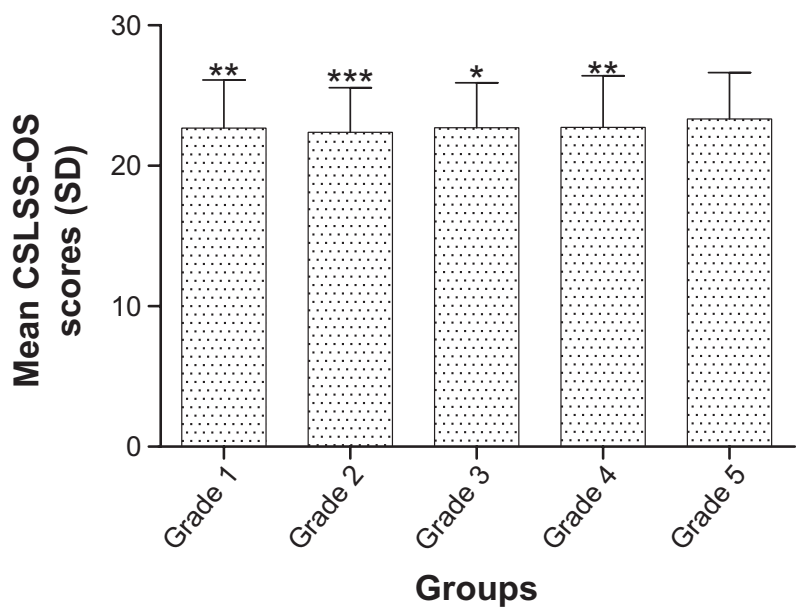

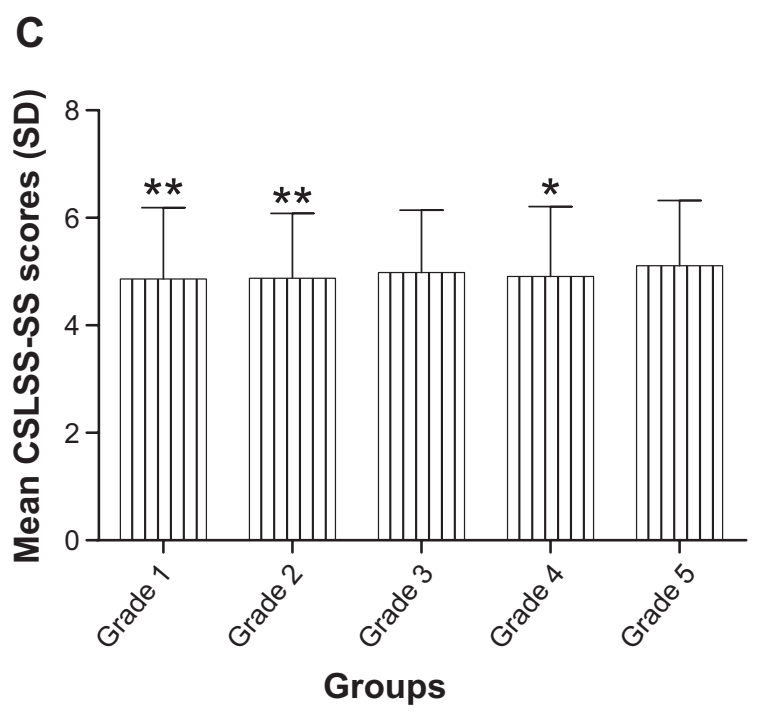

Figure 2 Comparisons of CSLSS total scores and subscale scores among five groups.

Notes: (A) CSLSS total scores. CSLSS total scores of grade 5 compared to other grades, $* P<0.05$; $* * P<0.0$ I; $* * * P<0.00$ I. (B) CSLSS-OS scores of grade 5 compared to other grades, $* P<0.05$; $* * P<0.01$; $* * * P<0.001$. (C) CSLSS-SS scores of grade 5 compared to other grades, $* P<0.05 ; * * P<0.01$.

Abbreviations: CSLSS, Life Satisfaction Scales Applicable to College Students; CSLSS-OS, CSLSS Objective Satisfaction subscale; CSLSS-SS, CSLSS Subjective Satisfaction subscale; SD, standard deviation. 
$F(4,2,081)=84.571, P<0.001$. The TAS-F1 (Figure 4B) was similar to the TAS total: $F(4,2,081)=20.661, P<0.001$. The TAS-F2 (Figure 4C) represents difficulties in describing or communicating feelings, and the scores of grade 1 and grade 2 were significantly higher compared with the other groups: $F(4,2,081)=19.436, P<0.001$. The grade 1 TAS-F3 (Figure 4D) scores were the highest, whereas the grade 5 scores were the lowest across the five grades: $F(4,2,081)=184.459, P<0.001$. These results suggested that the students in grade 1 not only had more difficulty in identifying emotion, but also in describing emotion and externally oriented thinking.

\section{Correlates of health anxiety}

The correlations are presented in Table 2. There was a significant negative correlation between the SHAI total score and each of the CSLSS total score, the CSLSS-OS, and the CSLSS-SS $(P<0.01)$. In terms of the TAS-20, there was a significant positive correlation between the SHAI total score and each of the TAS total score, the TAS-F1, the TAS-F2, and the TAS-F3 $(P<0.01)$. In the correlation between health anxiety and personality, the results suggested that the SHAI total score was not significantly correlated with the EPQ-E, but there was a significant positive correlation between the
A

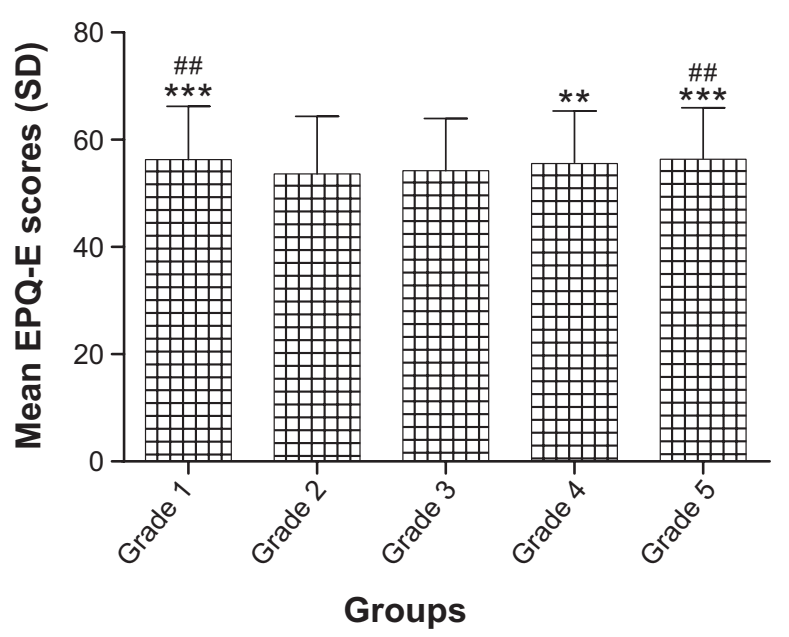

C

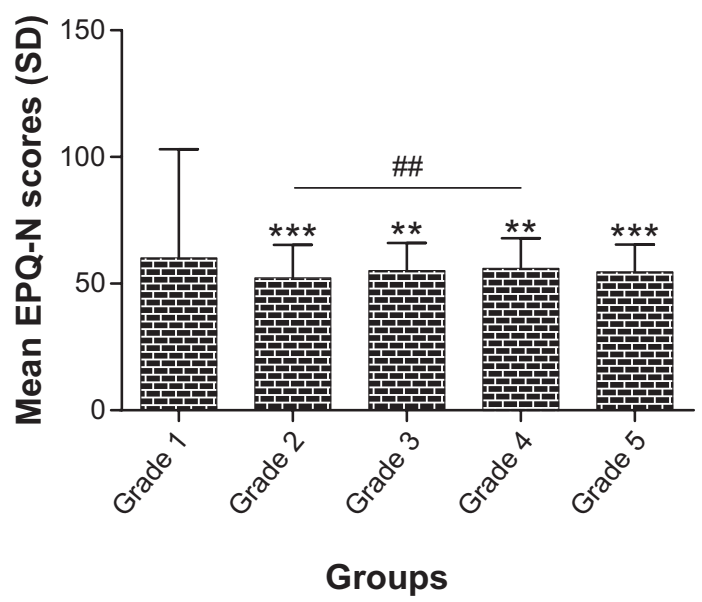

B

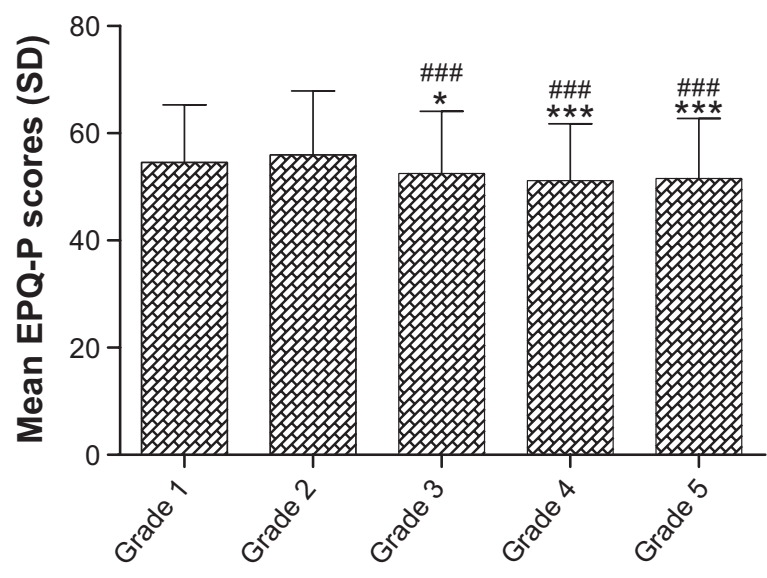

Groups

D

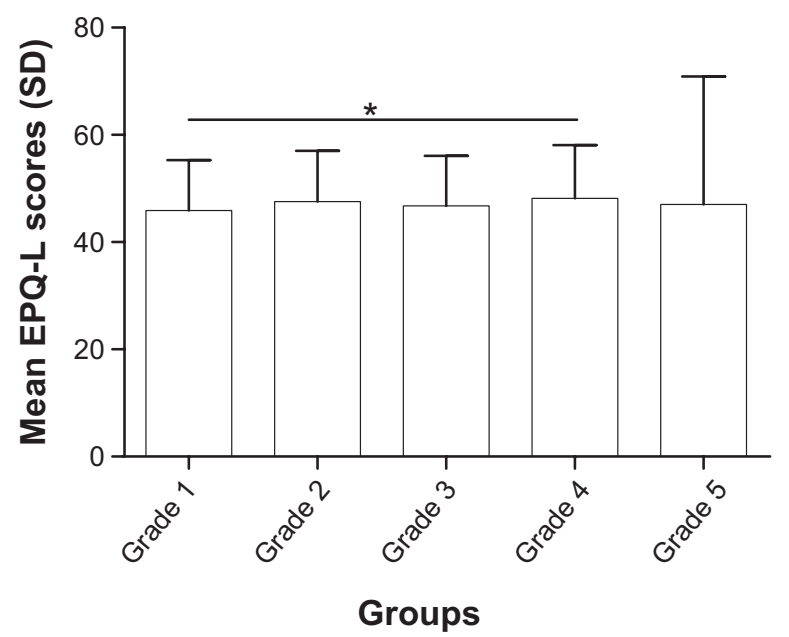

Figure 3 Comparisons of EPQ scores among five grades.

Notes: (A) EPQ-E scores. EPQ-E scores of grade 2 compared to other groups, $* * P<0.01$; $* * * P<0.00 I$; EPQ-E scores of grade 3 compared to other grades,

$\ldots P<0.0$ I. (B) EPQ-P scores. EPQ-P scores of grade I compared to other grades, $* P<0.05 ; * * * P<0.00 I$; EPQ-P scores of grade 2 compared to other grades, ${ }^{\prime} P<0.001$. (C) EPQ-N scores. EPQ-N scores of grade I compared to other grades, ${ }^{* * P}<0.01$; ${ }^{* * * P}<0.00 \mathrm{I}$. EPQ-N scores of grade 2 compared to grade 4 , ${ }^{\#} P<0.01$. (D) EPQ-L scores. EPQ-L scores of grade I compared to grade 4, ${ }^{*} P<0.05$.

Abbreviations: EPQ, Eysenck Personality Questionnaire; EPQ-E, EPQ Extraversion subscale; EPQ-L, EPQ Truthfulness subscale; EPQ-N, EPQ Neuroticism subscale; EPQ-P, EQP Psychoticism subscale; SD, standard deviation. 
A

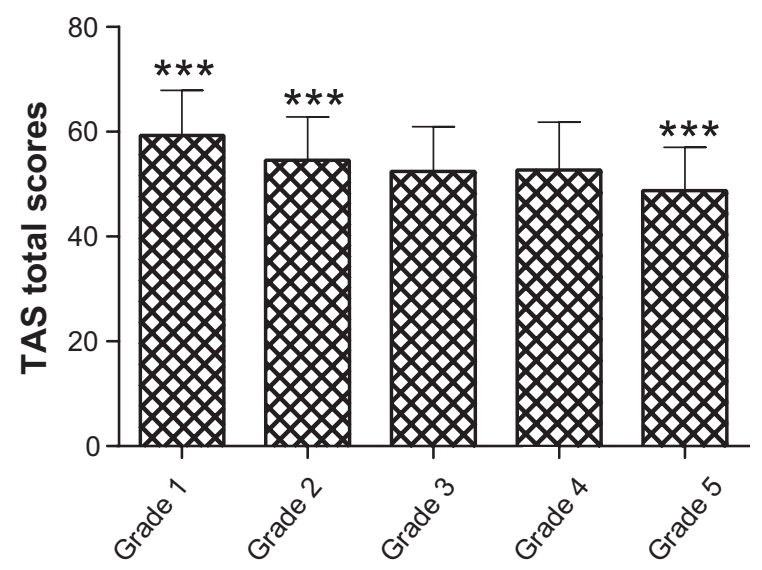

Groups

C

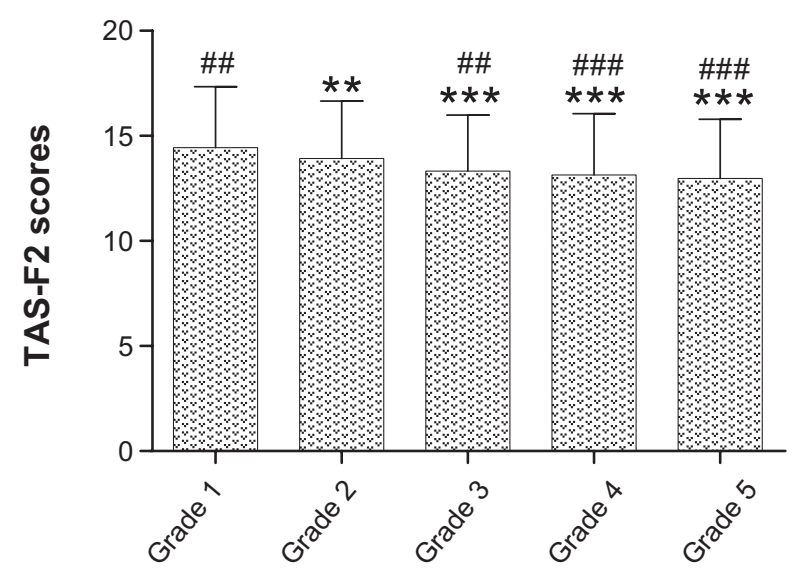

Groups
B

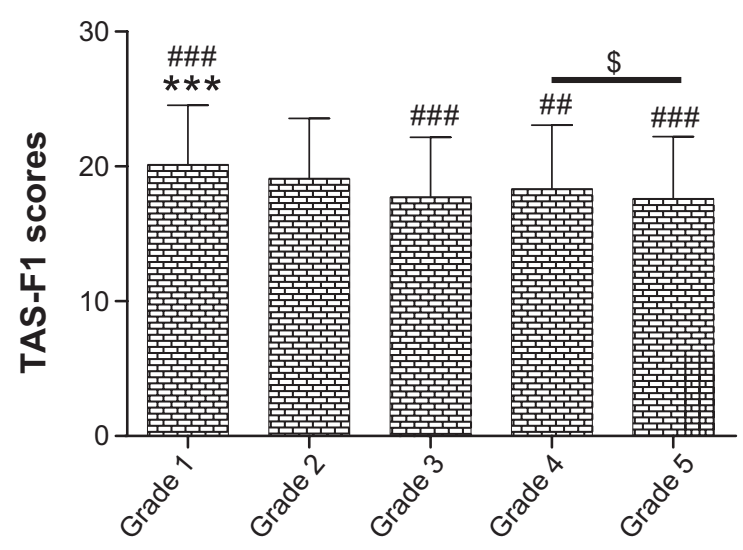

Groups

D

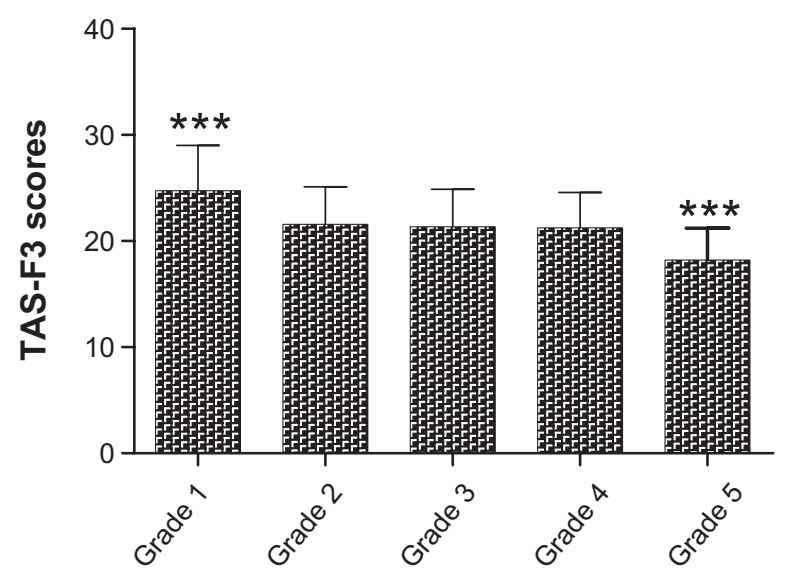

Groups

Figure 4 Comparisons of TAS-20 total scores and subscale scores among five groups.

Notes: (A) TAS-20 total scores. TAS-20 total scores of grade I, grade 2, and grade 5 compared to other grades, each $* * * P<0.001$. (B) TAS-FI scores of grade I compared

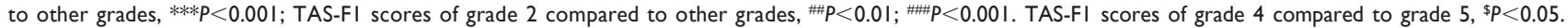
(C) TAS-F2 scores of grade I compared to other grades, ${ }^{* * P}<0.0 \mathrm{I}$; $* * * P<0.00 \mathrm{I}$; TAS-F2 scores of grade 2 compared to other grades, ${ }^{\# P}<0.0 \mathrm{I}$; $P<0.00 \mathrm{I}$. (D) TAS-F3 scores of grade I and grade 5 compared to other grades, each $* * * P<0.001$.

Abbreviations: TAS-20, Toronto Alexithymia Scale; TAS-FI, difficulties identifying and distinguishing among feelings; TAS-F2, difficulties describing or communicating feelings; TAS-F3, externally oriented thinking.

SHAI total score and the EPQ-P and the EPQ-N. The correlations between the SHAI-IL and other measures were similar to the SHAI total. The SHAI-NC had some differences, such as it was not significantly correlated with the TAS-F3, but there was a significant negative correlation between the SHAI-NC and the EPQ-E.

\section{Hierarchical regression analyses}

The variance inflation factor was calculated to test for multicollinearity among the predictors and was within normal ranges for all predictors (variance inflation factor $=1.041-1.909$ ). Further examination of the data also indicated the assumptions of both linearity and homoscedasticity were met.
As shown in Table 3, hierarchical liner regression analyses were used to examine whether health anxiety was predicted by demographic or psychological variables. In this model, age, being an only child, and residence were entered as independent variables in the first step: $F \Delta(3,2,082)=2.787, P<0.05, \Delta R^{2}=0.004$. In the second step, the CSLSS-OS and the CSLSS-SS were added to the model: $F \Delta(2,2,080)=36.964, P<0.001, \Delta R^{2}=0.078$. In the third step, the three factors of the EPQ were entered as independent variables: $F \Delta(3,2,077)=29.867, P<0.001, \Delta R^{2}=0.021$. In the fourth step, the three factors of the TAS-20 were also added to the model: $F \Delta(3,2,074)=38.301, P<0.001, \Delta R^{2}=0.066$. The four steps were all statistically significant. The results 
Table 2 Pearson correlation coefficients between SHAI total and subscale scores with related measures

\begin{tabular}{llll}
\hline & SHAI total & SHAI-IL & SHAI-NC \\
\hline SHAI-IL & $0.946^{* *}$ & - & - \\
SHAI-NC & $0.666^{* *}$ & $0.413^{* *}$ & - \\
CSLSS total & $-0.268^{* *}$ & $-0.258^{* *}$ & $-0.164^{* *}$ \\
CSLSS-OS & $-0.237^{* *}$ & $-0.230^{* *}$ & $-0.142^{* *}$ \\
CSLSS-SS & $-0.237^{* *}$ & $-0.222^{* *}$ & $-0.152^{* *}$ \\
EPQ-E & -0.039 & -0.024 & $-0.06 I^{* *}$ \\
EPQ-P & $0.100^{* *}$ & $0.093^{* *}$ & $0.049^{*}$ \\
EPQ-N & $0.176^{* *}$ & $0.165^{* *}$ & $0.123^{* *}$ \\
TAS-20 total & $0.262^{* *}$ & $0.255^{* *}$ & $0.159^{* *}$ \\
TAS-FI & $0.318^{* *}$ & $0.309^{* *}$ & $0.188^{* *}$ \\
TAS-F2 & $0.252^{* *}$ & $0.241^{* *}$ & $0.161^{* *}$ \\
TAS-F3 & $0.052^{* *}$ & $0.054^{*}$ & 0.032 \\
\hline
\end{tabular}

Notes: $* P<0.05 ; * * P<0.01$.

Abbreviations: SHAl, Short Health Anxiety Inventory; SHAIIL, SHAI Illness Likelihood subscale; SHAI-NC, SHAI Negative Consequences subscale; CSLSS, Life Satisfaction Scales Applicable to College Students; CSLSS-OS, CSLSS Objective Satisfaction subscale; CSLSS-SS, CSLSS Subjective Satisfaction subscale; TAS-20, Toronto Alexithymia Scale; TAS-FI, difficulties identifying and distinguishing among feelings; TAS-F2, difficulties describing or communicating feelings; TAS-F3, externally oriented thinking; EPQ-E, Eysenck Personality Questionnaire Extraversion subscale; EPQ-P, Eysenck Personality Questionnaire Psychoticism subscale; EPQ-N, Eysenck Personality Questionnaire Neuroticism subscale.

identified age, the OS and SS factors of the CSLSS, the neuroticism factor of personality, and the three factors of alexithymia as the determinants of the health anxiety total score in the final model.

The associations between the predictor variables and health anxiety were consistent with our hypotheses, such that these associations were in the predicted direction. Thus, from this study, we determined that life satisfaction, alexithymia, and individual personality may influence health anxiety.

\section{Discussion}

In this study, we investigated health anxiety using the SHAI. The comparison of the SHAI-IL suggested that the students in grade 2 had a greater fear of becoming ill compared with the other grades. We suggest that students in grade 2 begin to learn about diseases, but do not yet have sufficient knowledge of them. Thus, they may have feelings of having some symptoms and diseases which they recently learned about in books. In consideration of the initial clinical practice, which is perhaps the most stressful period in a student's education, ${ }^{32}$ the students in grade 5 also had higher SHAI-IL scores. For the SHAI-NC, which is another factor of the SHAI and which represents fearing the negative consequences of becoming ill, the first-year junior college students had significantly lower scores compared with the other groups. First-year students do not have sufficient medical knowledge; thus, they do not have a greater utilization of health professional services. On the contrary, they could have misunderstandings to some symptoms which were common in daily life.A previous study of medical students found that stress was significantly higher among students in the second and third Bachelor of Medicine and Bachelor of Surgery compared with those in the first Bachelor of Medicine and Bachelor of Surgery level..$^{39}$ We suggest that, because the students in their first year had less stress, their health anxiety of negative consequences was better.

The present study provides promising evidence that health anxiety in students is associated with life satisfaction, alexithymia, and personality. The results are consistent with our hypotheses. However, our expected influencing factors only predicted $16.9 \%$ of health anxiety, which indicates that the health anxiety of nursing students was impacted by them. As early as 1990 , Parkerson et $\mathrm{al}^{40}$ found that the students who were very satisfied with life had fewer symptoms of depression and anxiety, and their physical and mental health was better compared with individuals with low satisfaction. This conclusion is consistent with our results. Regardless of the SHAI total, the SHAI-IL and the SHAI-NC were negatively correlated with the CSLSS total and its two subscales. Therefore, the students who were satisfied with their lives, whether measured as OS or SS, had less health anxiety. Furthermore, the two subscales of the CSLSS predicted $7.8 \%$ of health anxiety.

A previous study suggested that patients with hypochondriasis carry a heavy burden of personality pathology. ${ }^{41}$ The present study found that psychoticism was positively correlated with health anxiety, which indicates that the students who were antisocial had less health anxiety. At the same time, neuroticism was also positively correlated with health anxiety. Neuroticism has been associated with a vulnerability to depression in initially non-depressed individuals, and numerous studies have found that neuroticism is a powerful variable for unipolar depression. ${ }^{42}$ Psychoticism and neuroticism predicted $2.1 \%$ of health anxiety.

There is some suggestion that mood conditions and somatic symptoms can play intervening roles in the etiology of health anxiety. Moreover, individuals with alexithymia are less able to cope properly with stressful situations and therefore experience more somatic symptoms when facing stress and anxiety. ${ }^{24}$ Thus, the present study examined the powers of the alexithymia subscales to predict health anxiety. The results revealed that alexithymia is an important predicted factor of health anxiety, and the three subscales of TAS-20 predicted $6.6 \%$ of health anxiety. A previous study found that individuals who had high levels of alexithymia reported a marginally significant increase in respiratory infection and symptoms of anxiety and depression. ${ }^{43}$ In our study, alexithymia was positively correlated with health anxiety. 
Table 3 Regression analysis examining predictors of SHAI total

\begin{tabular}{|c|c|c|c|c|c|c|c|}
\hline Variable & B & SE B & $\beta$ & $\mathbf{t}$ & Zero-order $\boldsymbol{r}$ & $\mathbf{R}^{2}$ & $\mathbf{F}$ \\
\hline Step I & & & & & & 0.004 & $2.787^{*}$ \\
\hline Age & -0.005 & 0.071 & -0.002 & -0.070 & 0.008 & & \\
\hline Only child (yes=l; no=2) & 0.714 & 0.293 & 0.054 & $2.437^{*}$ & 0.059 & & \\
\hline Residence (urban $=\mathrm{I}$; rural $=2$ ) & 0.280 & 0.260 & 0.024 & 1.076 & 0.034 & & \\
\hline Step 2 & & & & & & 0.082 & $36.964 * * *$ \\
\hline Age & 0.070 & 0.069 & 0.022 & 1.018 & 0.008 & & \\
\hline Only child (yes=I; no=2) & 0.583 & 0.282 & 0.044 & $2.070 *$ & 0.059 & & \\
\hline Residence (urban=I; rural=2) & 0.268 & 0.250 & 0.023 & 1.072 & 0.034 & & \\
\hline CSLSS-OS & -0.272 & 0.039 & -0.164 & $-7.002 * * *$ & -0.237 & & \\
\hline CSLSS-SS & -0.748 & 0.106 & -0.166 & $-7.085^{* * *}$ & -0.237 & & \\
\hline Step 3 & & & & & & 0.103 & 29.867 **** \\
\hline Age & 0.109 & 0.069 & 0.034 & 1.585 & 0.008 & & \\
\hline Only child (yes=l; no=2) & 0.480 & 0.279 & 0.036 & 1.721 & 0.059 & & \\
\hline Residence (urban $=\mathrm{I}$; rural $=2$ ) & 0.243 & 0.248 & 0.021 & 0.979 & 0.034 & & \\
\hline CSLSS-OS & -0.246 & 0.039 & -0.148 & $-6.340 * * *$ & -0.237 & & \\
\hline CSLSS-SS & -0.688 & 0.105 & -0.148 & $-6.359 * * *$ & -0.237 & & \\
\hline EPQ-E & -0.012 & 0.012 & -0.021 & -0.990 & -0.039 & & \\
\hline EPQ-P & 0.026 & 0.011 & 0.052 & $2.458^{*}$ & 0.100 & & \\
\hline EPQ-N & 0.036 & 0.006 & 0.135 & $6.348 * * *$ & 0.176 & & \\
\hline Step 4 & & & & & & 0.169 & $38.301 * * * *$ \\
\hline Age & 0.148 & 0.072 & 0.046 & $2.056 *$ & 0.008 & & \\
\hline Only child (yes=I; no=2) & 0.416 & 0.269 & 0.032 & 1.545 & 0.059 & & \\
\hline Residence (urban=l; rural=2) & 0.185 & 0.240 & 0.016 & $0.77 \mid$ & 0.034 & & \\
\hline CSLSS-OS & -0.198 & 0.038 & -0.119 & $-5.249 * * *$ & -0.237 & & \\
\hline CSLSS-SS & -0.588 & 0.102 & -0.131 & $-5.249 * * *$ & -0.237 & & \\
\hline EPQ-E & -0.005 & 0.011 & -0.009 & -0.419 & -0.039 & & \\
\hline EPQ-P & 0.015 & 0.010 & 0.031 & 1.495 & 0.100 & & \\
\hline EPQ-N & 0.024 & 0.006 & 0.089 & $4.270 * * *$ & 0.176 & & \\
\hline TAS-FI & 0.279 & 0.033 & 0.231 & $8.412 * * *$ & 0.318 & & \\
\hline TAS-F2 & 0.140 & 0.054 & $0.07 I$ & $2.579 * *$ & 0.252 & & \\
\hline TAS-F3 & -0.094 & 0.032 & -0.069 & $-2.952^{* *}$ & 0.052 & & \\
\hline
\end{tabular}

Notes: $* * * P<0.001$, $* * P<0.01, * P<0.05$.

Abbreviations: SHAI, Short Health Anxiety Inventory; CSLSS-OS, Life Satisfaction Scales Applicable to College Students Objective Satisfaction subscale; CSLSS-SS, Life Satisfaction Scales Applicable to College Students Subjective Satisfaction subscale; EPQ-E, Eysenck Personality Questionnaire Extraversion subscale; EPQ-P, Eysenck Personality Questionnaire Psychoticism subscale; EPQ-N, Eysenck Personality Questionnaire Neuroticism subscale; B, statistical variable in regression (coefficient); TAS, Toronto Alexithymia Scale; TAS-FI, difficulties identifying and distinguishing among feelings; TAS-F2, difficulties describing or communicating feelings; TAS-F3, externally oriented thinking.

Moreover, in the base of the cognitive behavior model of anxiety disorders, it is worth considering that alexithymia may affect health anxiety. A study has suggested that expressive writing is a good method of reducing health care utilization in healthy samples. ${ }^{44}$ Therefore, individuals who have health anxiety can also try expressive writing to reduce the severity of their anxiety.

The current results should be incorporated in clinical practice. Colleges should pay more attention to nursing students through using varying strategies in different grades because of the different psychological problems. Correct psychological education should persist throughout all college years. When nursing students begin to acquire medical knowledge, professors should help them understand the diseases, so that they have correct cognitions and the prevalence of health anxiety can decline. The dimensional construct of health anxiety results in complex inducements. In the combination of the imbalance in the gender ratio of nursing students, colleges should enhance nursing skills and interpersonal relationships in order to respond to clinical training and then reduce health anxiety.

\section{Limitations and future directions}

A limitation of this study is that we recruited students in one college rather than multiple colleges. The results will be affected by college-specific factors, such as the teachers, learning environment, and course arrangement. Furthermore, when we investigated the nursing students, we did not control for depression, other types of anxiety disorders, or psychosis. The results could change after controlling for these factors. Moreover, there was an imbalance in the female-to-male ratio. Finally, the relationship between health anxiety and other factors should be investigated further because health anxiety is affected by complex factors. Diagnostic criteria of health anxiety should be proposed to contribute to clinical treatment. 


\section{Conclusion}

Health anxiety is common not only in patients who have a physical disease or psychological disorders, but also in the normal population. In this study, which investigated nursing students, the findings show that health anxiety was negatively correlated with life satisfaction. Thus, with the improvement of life satisfaction, the health anxiety of nursing students would decline. Personality and alexithymia were positively correlated with health anxiety, but the correlation coefficients were low. In addition, objective and subjective life satisfaction, neuroticism, and the three aspects of the TAS-20 could be predictors of health anxiety. These factors accounted for $16.9 \%$ of the nursing students' health anxiety. On the basis of this evidence, life satisfaction, neuroticism of personality, and alexithymia were the influencing factors of health anxiety in Chinese nursing students.

\section{Disclosure}

The authors report no conflicts of interest in this work.

\section{References}

1. Hadjistavropoulos HD, Janzen JA, Kehler MD, Leclerc JA, Sharpe D, Bourgault-Fagnou MD. Core cognitions related to health anxiety in self-reported medical and non-medical samples. J Behav Med. 2012;35: 167-178.

2. Ferguson E. A taxometric analysis of health anxiety. Psychol Med. 2009;39:277-285.

3. Fink P, Ørnbøl E, Christensen KS. The outcome of health anxiety in primary care. A two-year follow-up study on health care costs and self-rated health. PLoS One. 2010;5:e9873.

4. Sunderland M, Newby JM, Andrews G. Health anxiety in Australia: prevalence, comorbidity, disability and service use. Br J Psychiatry. 2013; 202:56-61.

5. Tyrer P, Cooper S, Crawford M, et al. Prevalence of health anxiety problems in medical clinics. J Psychosom Res. 2011;71:392-394.

6. Reisbig AM, Danielson JA, Wu TF, et al. A study of depression and anxiety, general health, and academic performance in three cohorts of veterinary medical students across the first three semesters of veterinary school. J Vet Med Educ. 2012;39:341-358.

7. Wedig MM, Frankenburg FR, Bradford Reich D, Fitzmaurice G, Zanarini MC. Predictors of suicide threats in patients with borderline personality disorder over 16 years of prospective follow-up. Psychiatry Res. 2013;208:252-256.

8. Whitton SW, Kuryluk AD. Relationship satisfaction and depressive symptoms in emerging adults: cross-sectional associations and moderating effects of relationship characteristics. J Fam Psychol. 2012;26: 226-235.

9. Divney AA, Sipsma H, Gordon D, Niccolai L, Magriples U, Kershaw T. Depression during pregnancy among young couples: the effect of personal and partner experiences of stressors and the buffering effects of social relationships. J Pediatr Adolesc Gynecol. 2012;25:201-207.

10. Richards HS, Jenkinson E, Rumsey N, et al. The psychological wellbeing and appearance concerns of patients presenting with ptosis. Eye (Lond). 2014;28(3):296-302.

11. Hussain R, Guppy M, Robertson S, Temple E. Physical and mental health perspectives of first year undergraduate rural university students. BMC Public Health. 2013;13:848.

12. Kraus MW, Piff PK, Mendoza-Denton R, Rheinschmidt ML, Keltner D. Social class, solipsism, and contextualism: how the rich are different from the poor. Psychol Rev. 2012;119:546-572.
13. Widiger TA, Simonsen E. Alternative dimensional models of personality disorder: finding a common ground. J Pers Disord. 2005;19: 110-130.

14. Ciarrochi J, Heaven PCL. Longitudinal examination of the impact of Eysenck's psychoticism dimension on emotional well-being in teenagers. Pers Individ Dif. 2007;42:597-608.

15. Ciarrochi J, Heaven PCL. A longitudinal study into the link between adolescent personality and peer-rated likeability and adjustment: evidence of gender differences. J Res Pers. 2009;43:978-986.

16. Bech P, Lunde M, Møller SB. Eysenck's two big personality factors and their relationship to depression in patients with chronic idiopathic pain disorder: a clinimetric validation analysis. ISRN Psychiatry. 2012;2012: 140458.

17. McKinnon AC, Van Oudenhove L, Tack J, Jones M. The association of personality, appraisal, catastrophising and vigilance with gastrointestinal symptom-specific anxiety. J Health Psychol. Epub 2013 Oct 22.

18. Nemiah JC, Sifneos PE. Psychosomatic illness: a problem in communication. Psychother Psychosom. 1970;18:154-160.

19. Reker M, Ohrmann P, Rauch AV, et al. Individual differences in alexithymia and brain response to masked emotion faces. Cortex. 2010;46: 658-667.

20. Borsci G, Boccardi M, Rossi R, et al. Alexithymia in healthy women: a brain morphology study. J Affect Disord. 2009;114:208-215.

21. Bagby RM, Quilty LC, Taylor GJ. Are there subtypes of alexithymia? Pers Individ Dif. 2009;47:413-418.

22. Zunhammer M, Eberle H, Eichhammer P, Busch V. Somatic symptoms evoked by exam stress in university students: the role of alexithymia, neuroticism, anxiety and depression. PLoS One. 2013;8(12):e84911.

23. Deng Y, Ma X, Tang Q. Brain response during visual emotional processing: an fMRI study of alexithymia. Psychiatry Res. 2013;213: 225-229.

24. Shahidi S, Molaie A, Dehghani M. Relationship between health anxiety and alexithymia in an Iranian sample. Procedia Soc Behav Sci. 2012;46: 591-595.

25. Reiser SJ, McMillan KA, Wright KD, Asmundson GJ. Adverse childhood experiences and health anxiety in adulthood. Child Abuse Negl. 2014;38(3):407-413.

26. Alberts NM, Hadjistavropoulos HD. Parental illness, attachment dimensions, and health beliefs: testing the cognitive-behavioural and interpersonal models of health anxiety. Anxiety Stress Coping. 2014;27(2): 216-228.

27. Gerolimatos LA, Edelstein BA. Anxiety-related constructs mediate the relation between age and health anxiety. Aging Ment Health. 2012;16: 975-982.

28. Rachman S. Health anxiety disorders: a cognitive construal. Behav Res Ther. 2012;50:502-512.

29. Baghurst T, Kelley BC. An examination of stress in college students over the course of a semester. Health Promot Pract. 2014;15(3): $438-447$.

30. Gramstad TO, Gjestad R, Haver B. Personality traits predict job stress, depression and anxiety among junior physicians. BMC Med Educ. 2013;13:150.

31. Chernomas WM, Shapiro C. Stress, depression, and anxiety among undergraduate nursing students. Int J Nurs Educ Scholarsh. 2013;10.

32. Salkovskis PM, Rimes KA, Warwick HM, Clark DM. The Health Anxiety Inventory: development and validation of scales for the measurement of health anxiety and hypochondriasis. Psychol Med. 2002;32: 843-853.

33. Alberts NM, Sharpe D, Kehler MD, Hadjistavropoulos HD. Health anxiety: Comparison of the latent structure in medical and non-medical samples. J Anxiety Disord. 2011;25:612-614.

34. Alberts NM, Hadjistavropoulos HD, Jones SL, Sharpe D. The Short Health Anxiety Inventory: a systematic review and meta-analysis. J Anxiety Disord. 2013;27:68-78.

35. Wang YZ. Life satisfaction scales applicable to college students. In: Zhang ZJ, editor. Behavioral Medicine Inventory Manual. Vol 17. Beijing. The Chinese Medicine Electronic Audio and Video Publishing House; 2005:420-423. Chinese 
36. Yuan YG, Shen XH, Zhang XR, et al. The reliability and validity of Toronto Alexithymia Scale (TAS-20). Sichuan Ment Health. 2003;16: 25-27. Chinese.

37. Zhang ZJ. Eysenck Personality Quesionnaire. In: Zhang ZJ, editor. Behavioral Medicine Inventory Manual. Vol 17. Beijing. The Chinese Medicine Electronic Audio and Video Publishing House; 2005:36-38. Chinese.

38. Aaseth K, Grande RB, Leiknes KA, Benth JŠ, Lundqvist C, Russell MB. Personality traits and psychological distress in persons with chronic tension-type headache. The Akershus study of chronic headache. Acta Neurol Scand. 2011;124:375-382.

39. Inam SB. Anxiety and depression among students of a medical college in Saudi Arabia. Int J Health Sci (Qassim). 2007;1:295-300.

40. Parkerson GR Jr, Broadhead WE, Tse CK. The health status and life satisfaction of first-year medical students. Acad Med. 1990;65: 586-588.
41. Fallon BA, Harper KM, Landa A, et al. Personality disorders in hypochondriasis: Prevalence and comparison with two anxiety disorders. Psychosomatics. 2012;53:566-574.

42. Lam D, Smith N, Checkley S, Rijsdijk F, Sham P. Effect of neuroticism, response style and information processing on depression severity in a clinically depressed sample. Psychol Med. 2003;33:469-479.

43. Niles AN, Haltom KE, Mulvenna CM, Lieberman MD, Stanton AL. Randomized controlled trial of expressive writing for psychological and physical health: the moderating role of emotional expressivity. Anxiety Stress Coping. 2014;27(1):1-17.

44. Harris AH. Does expressive writing reduce health care utilization? A meta-analysis of randomized trials. J Consult Clin Psychol. 2006; $74: 243-252$.

\section{Publish your work in this journal}

Neuropsychiatric Disease and Treatment is an international, peerreviewed journal of clinical therapeutics and pharmacology focusing on concise rapid reporting of clinical or pre-clinical studies on a range of neuropsychiatric and neurological disorders. This journal is indexed on PubMed Central, the 'PsycINFO' database and CAS, and is the official journal of The International Neuropsychiatric Association (INA). The manuscript management system is completely online and includes a very quick and fair peer-review system, which is all easy to use. Visit http://www.dovepress.com/testimonials.php to read real quotes from published authors.

\footnotetext{
Submit your manuscript here: http://www.dovepress.com/neuropsychiatric-disease-and-treatment-journal
} 